\title{
Vanishing Aortic Thrombus
}

\author{
Azad Abdul Salamª, , Manoj Ravia ${ }^{\text {a }}$ T. Govindan Unni ${ }^{\mathrm{a}}$
}

\begin{abstract}
Acute thrombosis of ascending aorta and arch is a relatively rare entity. Multiple rare etiologies are described in literature. We report a rare case of acute thrombosis of aorta in a young obese lady with metabolic syndrome.
\end{abstract}

Keywords: Aortic thrombosis; Metabolic syndrome; Thrombophilic states

\section{Introduction}

Cardiovascular thromboembolism is a well-defined entity with multiple etiopathogenesis. Of all the causes, the most common source of systemic thromboembolism is the heart. Development of a thrombus in the ascending aorta or its arch in a grossly normal aorta is rare. We present a case report of rapidly disappearing large thrombus in the proximal aorta.

\section{Case Report}

A 39-year-old woman was presented to the casualty with a history of left lower limb pain for $2 \mathrm{~h}$ followed by weakness of left lower limb and then rest angina and profuse sweating for $2 \mathrm{~h}$. All symptoms occurred over a time period of $6-7 \mathrm{~h}$. The patient also had irrelevant speech for $1 \mathrm{~h}$ prior to admission. Patient was diabetic for past 1 year on irregular medication. There was no history of prior similar complaints. There was no prior history of hypertension, coronary artery disease, stroke, any thrombotic episodes, tuberculosis, or surgeries. She was unmarried and had regular menstrual cycles.

The patient was obese with a body mass index of $29 \mathrm{~kg} / \mathrm{m}^{2}$ and was slightly disoriented with irrelevant speech. Her pulse rate was $62 / \mathrm{min}$, regular. She had reduced pulse over left dor-

Manuscript submitted September 19, 2017, accepted October 17, 2017

aDepartment of Cardiology, Jubilee Mission Medical College \& Research Institute, Thrissur, Kerala, India

bCorresponding Author: Azad Abdul Salam, Department of Cardiology, Jubilee Mission Medical College \& Research Institute, Thrissur 680005, Kerala, India. Email: azadabdulsalam@gmail.com

doi: https://doi.org/10.14740/jmc2924w salis pedis and posterior tibial arteries. Her blood pressure on admission was 130/80 $\mathrm{mm} \mathrm{Hg}$ in right arm and 128/80 $\mathrm{mm} \mathrm{Hg}$ in left arm in supine position. Cardiovascular system examination otherwise was not significant. Neurological examination revealed flaccid paralysis of left lower limb with gross power $2 / 5$ at hip and knee, sluggish left ankle and knee jerks with left plantar extensor. Electrocardiogram (ECG) showed ST elevation in inferior leads. Two-dimensional echocardiography (2D echo) showed a large mass of $2 \times 2 \mathrm{~cm}$ in the aortic sinus close to the right coronary ostium which was partially mobile (Fig. 1). There was another $1 \times 1 \mathrm{~cm}$ thrombus in the descending aorta just distal to left subclavian artery which was also mobile (Fig. 2). She was immediately taken for computed tomography (CT) aortogram and CT of brain. Surprisingly CT aortogram did not show any mass/thrombus right from the heart to the middle of superficial femoral artery bilaterally (Fig. 3). Thrombus had probably embolized during transit. CT of brain showed a fresh infarct in the right frontal parasagittal region (Fig. 4). Repeat 2D echo did not reveal any thrombus. Meanwhile, patient became unstable and developed third degree atrioventricular (AV) block and cardiogenic shock. Patient was taken for coronary angiogram which showed a totally occluding thrombus in the distal right coronary artery. Patient underwent thrombosuction and primary plain old balloon angioplasty (POBA) under temporary pacemaker support with stabilization of cardiovascular parameters. Routine investigations revealed a normal hemogram and erythrocyte sedimentation rate was $40 \mathrm{~mm} / \mathrm{h}$. Renal and liver function tests were normal. Lipid profile revealed low high-density lipoproteins of $24 \mathrm{mg} / \mathrm{dL}$. Thyroid function test and serum homocysteine levels were normal. C-reactive protein (CRP) was highly raised $11.1 \mathrm{mg}$. Antinuclear antibodies and antiphospholipid antibodies were negative. Serologies of HIV, HbsAg, anti-HCV, and VDRL were negative. Prothrombin time and activated partial thromboplastin time were normal. Patient could not afford for other thrombophilia workup.

Patient was started on heparin and later switched to warfarin, double antiplatelets, statin, beta blocker and ACE inhibitors. Patient was discharged 1 week after admission.

\section{Discussion}

Thrombosis of abdominal aorta is a well-known entity with multiple etiologies. However, acute thrombosis of ascending aorta and arch is relatively rare. Aortic thrombosis is commonly secondary to atherosclerosis or embolic phenomenon, with bifurcation being the most common site. Hypercoagulable 


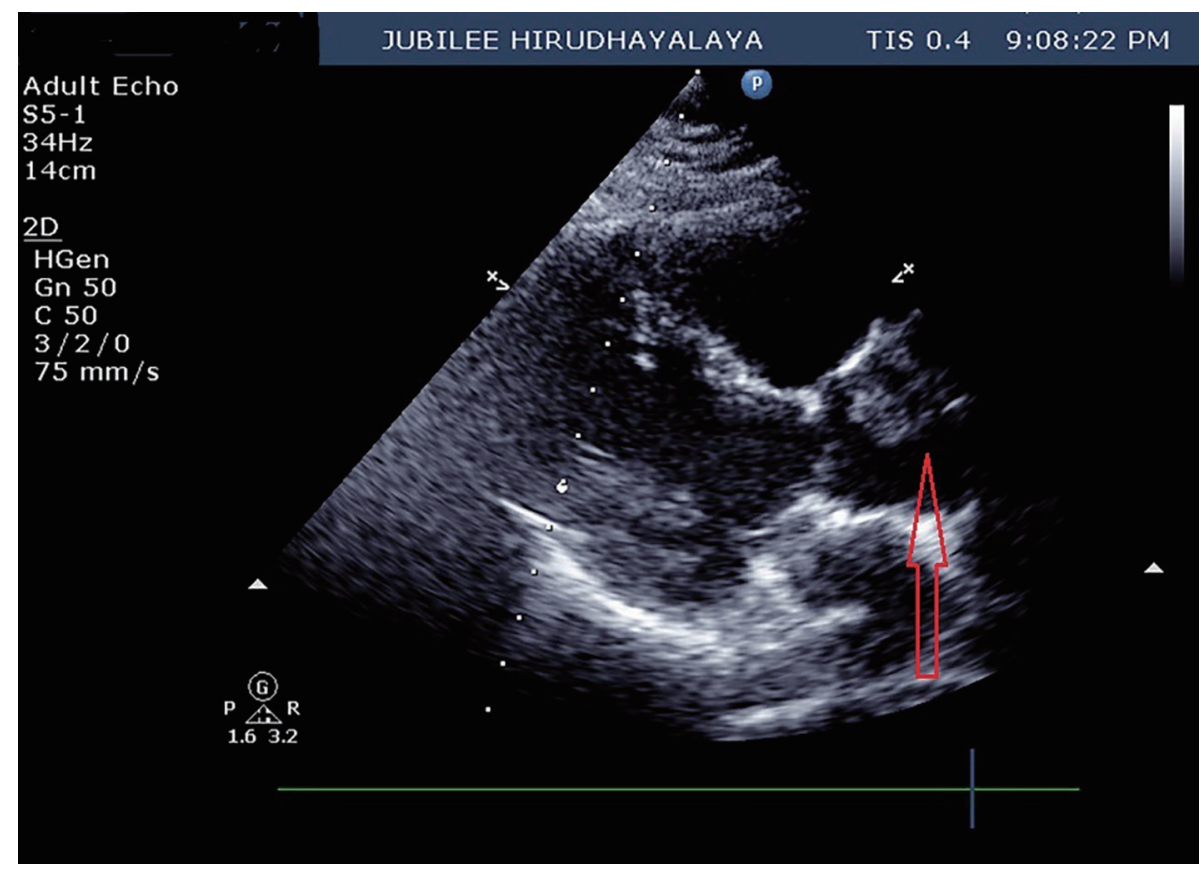

Figure 1. Parasternal long axis view on 2D echo showing thrombus (red arrow) at the level of aortic sinus.

states are less common but definite cause of venous thrombosis and rarely arterial thrombosis. Common factors that involve venous thromboembolism are factor $\mathrm{V}$ Leiden, prothrombin mutation GZ0210A and deficiencies of protein C, protein $S$, and antithrombin. Elevated levels of CRP, homocysteine and lipoprotein (a) are more likely to be involved with arterial thrombosis. Antiphospholipid antibodies are associated with both venous and arterial thrombosis [1].

The metabolic syndrome is a clinical entity of substantial heterogeneous traits represented by the co-occurrence of ab- dominal fat, impaired glucose tolerance, dyslipidemia (high triglycerides and low high-density lipoprotein (HDL) cholesterol levels) and hypertension. Metabolic syndrome is accompanied by important changes in the hemostatic system that may favor the development of thrombosis. Hyperactivity of platelets and hypercoagulability favor platelet and fibrin deposits, and hypo fibrinolysis due to the plasminogen activator inhibitor-1 (PAI1) excess prevents their elimination. Low HDL levels which accompany the metabolic syndrome could be involved in the thrombotic risk by increasing thrombin generation [2]. CRP

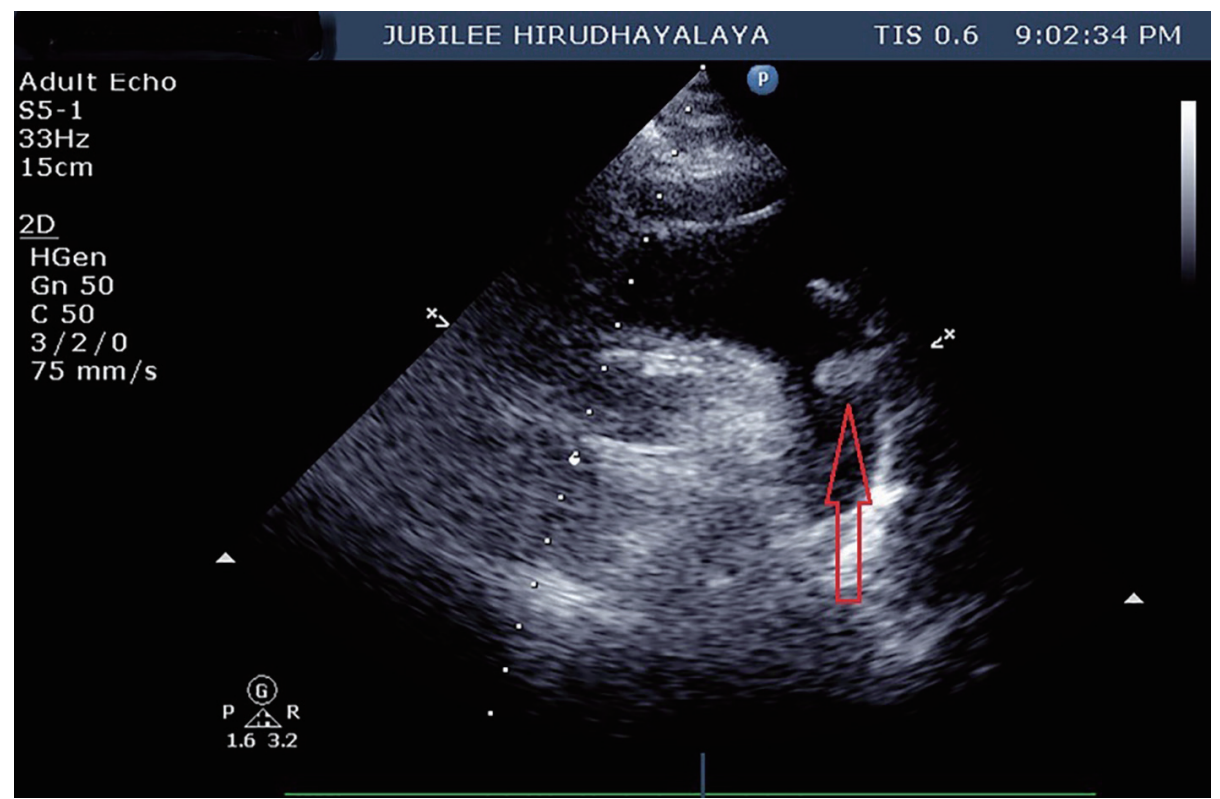

Figure 2. Suprasternal view on $2 \mathrm{D}$ echo showing arch of aorta and thrombus (red arrow) at the level of left subclavian artery. 


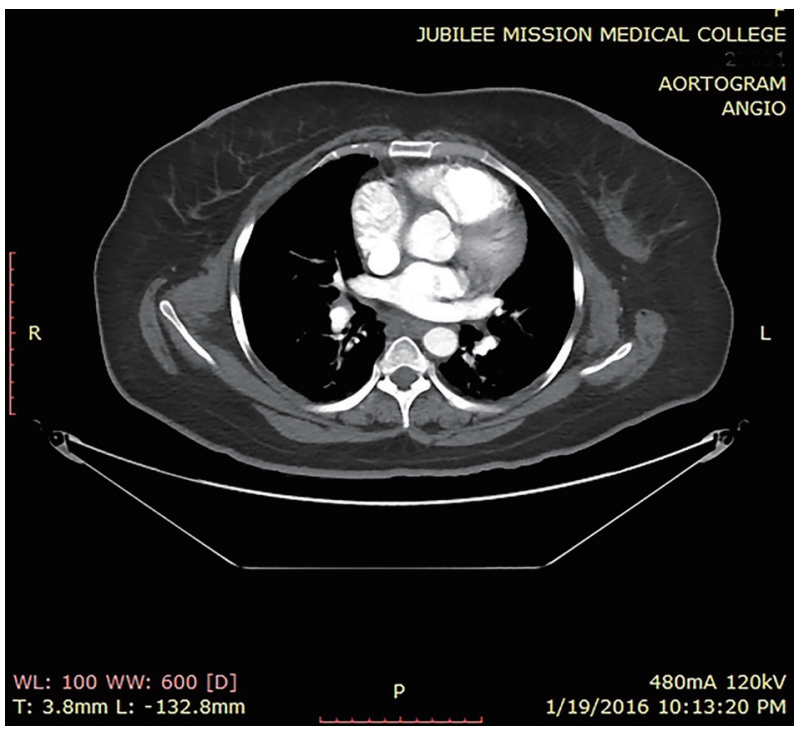

Figure 3. CT aortogram showing no thrombus in the aorta.

levels and PAI-1, a marker of atherothrombosis, are increased in subjects with the metabolic syndrome and diabetes. The in vitro study by Devaraj et al [3] showed the pivotal role for inflammation as assessed by increased CRP and increased PAI-1 levels, which seems to be the underpinning of atherothrombosis, especially in the metabolic syndrome and the diabetic state.

Our patient fulfilled the criteria for metabolic syndrome (waist circumference $96 \mathrm{~cm}$, diabetes mellitus, and low HDL $29 \mathrm{mg} / \mathrm{dL}$ ). We could not investigate for other thrombophilic states due to poor financial status of the patient. Diagnosis of aortic thrombosis warrants high index of suspicion especially when ischemia involves multiple organ systems over a short period of time. Even though our patient had a stormy course in hospital, she recovered fast and was able to resume her routine at 1 month of follow-up. This case points to the fact that metabolic syndrome may predispose to thrombosis at rare locations like aortic arch which has not been previously described in literature as far as our knowledge.

\section{Acknowledgments}

We extend our sincere gratitude to faculty members of Jubilee Center for Medical Research for editing this article.

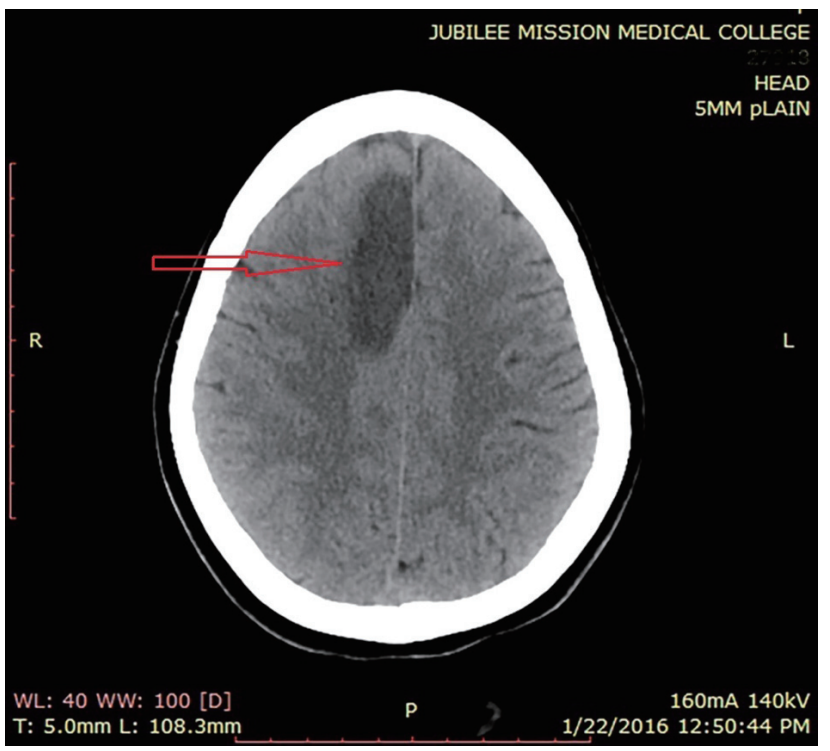

Figure 4. Plain CT of brain showing infarct (red arrow) at right frontal parasagittal region.

\section{Grant Support}

None.

\section{Conflict of Interest}

None.

\section{References}

1. Verma S, Yadav P, Srivastava D, Sharma SC, Rewari BB. Extensive aorto-arterial thrombosis. Indian Academy of Clinical Medicine. 2009;10(3):151-153.

2. Alessi MC, Juhan-Vague I. Metabolic syndrome, haemostasis and thrombosis. Thromb Haemost. 2008;99(6):9951000 .

3. Devaraj S, Xu DY, Jialal I. C-reactive protein increases plasminogen activator inhibitor-1 expression and activity in human aortic endothelial cells: implications for the metabolic syndrome and atherothrombosis. Circulation. 2003;107(3):398-404. 